\title{
Role of glucose-6-phosphate dehydrogenase for oxidative stress and apoptosis
}

\author{
Cell Death and Differentiation (2006) 13, 527-528. doi:10.1038/sj.cdd.4401807; published online 25 November 2005
}

Dear Editor,

Loss of function of glucose-6-phosphate dehydrogenase (G6PD) represents the most common inborn error of metabolism throughout the world which is known as G6PDdeficiency affecting an estimated 400 million people. ${ }^{1}$ Prolonged neonatal jaundice and hemolytic anemia are common clinical manifestations. Infections, ingestion of fava beans, and some drugs can trigger life-threatening hemolytic anemia. G6PD is the first enzyme of the pentose phosphate pathway that converts $\alpha$-D-glucose-6-phospate into D-glucono-1,5-lactone-6-phosphate and is involved in the generation of NADPH. As erythrocytes lack the citric acid cycle, the pentose phosphate shunt is the only source of NADPH. $\mathrm{NADPH}$ is required for the generation of reduced glutathione, which is important for the protection against oxidative damage. As the G6PD gene is located at the X-chromosome at $\mathrm{Xq} 28,{ }^{2}$ the disease is recessively inherited in males. Some 140 missense mutations leading to amino-acid substitutions and in a few cases base pair deletions that do not produce frame shifts are known. ${ }^{3}$ Few splicing mutations have been documented. ${ }^{4,5}$

Recently, Fico et al. ${ }^{6}$ stated that 'for the first time a role for G6PD in the protection from redox imbalance-induced apoptosis and necrosis has been clearly assigned' in a paper published by them. In fact, this has already been reported in 1995 by us. ${ }^{7}$ Since glutathione represents a cellular protectant against oxidative damage, which inhibits apoptosis, we hypothesized at that time that nucleated cells of G6PDdeficient patients should be more susceptible to DNA damage and apoptosis induced by oxidative stress. We reported that peripheral mononuclear cells (PBMC) from a G6PD-deficient male showed significantly higher apoptotic rates upon challenge by cytostatic drugs (daunorubicin), gamma-irradiation, or glucocorticoids (dexamethasone) than PBMC from healthy males. ${ }^{7}$ The induction of oxidative stress/generation of reactive oxygen species by all three agents has been reported. ${ }^{8-14}$ This implies that diverse ROS-generating agents affect white blood cells of G6PD-deficient patients leading to apoptosis due to insufficient protection from oxidative damage by reduced glutathione. We used PBMC of a German variant of G6PD deficiency, G6PD Aachen. This variant has originally been described by Kahn et al. ${ }^{15}$ The mutation in the G6PD Aachen variant has been determined by us previously. ${ }^{16} \mathrm{~A}$ mutation $1089 \mathrm{C}>\mathrm{G}$ results in a predicted amino-acid change 363 Asn $>$ Lys. The $1089 \mathrm{C}>\mathrm{G}$ point mutation is unique, but produces the identical amino-acid change found in another variant of G6PD deficiency, G6PD Loma Linda. The 363Asn > Lys exchange in G6PD Loma Linda is caused by a $1089 \mathrm{C}>\mathrm{A}$ mutation. ${ }^{17}$ Using the available three-dimensional structure of the human G6PD tetrameric protein complex, ${ }^{18}$ the location of the point mutation of amino acid 363 in G6PD Aachen is found at the surface of a monomer in close proximity to $\mathrm{NADP}^{+}$and more than $20 \AA$ away from the glucose-6-phosphate-binding site (data not shown). It can be speculated that this residue may be involved in NADP ${ }^{+}$binding that in turn is required for tetramer stability. ${ }^{18}$ Thus, Arg363 may be required to indirectly maintain the structural integrity of the functional unit. Replacing it with a positively charged Lys residue would lead to charge-charge repulsion between Lys363 and NADP ${ }^{+}$, thus affecting $\mathrm{NADP}^{+}$binding and tetramer formation.

In the same year as we initially reported on the increased induction of apoptosis in G6PD-deficient cells, another group reported that G6PD is essential for defense against oxidative stress using G6PD knockout mice. ${ }^{19}$ Knockout clones of embryonic stem cells were extremely sensitive to hydrogen peroxide and to the sulfydryl group oxidizing agent, diamide. In a subsequent study, we analyzed whether increased induction of apoptosis correlated with increased DNA damage in G6PD-deficient PBMC. ${ }^{20}$ In PBMC of three males of the G6PD Aachen variant and one G6PD-deficient male from another family coming from Iran, we found that UV light induced more DNA damage and more apoptosis than in PBMC of healthy male subjects. PBMC of heterozygote females showed intermediate rates of DNA damage and apoptosis. We concluded that increased DNA damage and apoptosis may be a result of increased oxidative stress in G6PD-deficient patients. UV radiation is known to induce DNA damage and apoptosis by the generation of reactive oxygen species. ${ }^{21-24}$ Interestingly, UV radiation induces hemolysis - a major complication in G6PD deficiency - which is reversible by the addition of glutathione. ${ }^{25}$ Recently, Mesbah-Namin et al. ${ }^{26}$ hypothesized that failure to detoxify hydrogen peroxide in G6PD-deficient leukocytes could induce primary DNA damage. Using the comet assay, these authors found that PBMC of 36 infants suffering from the Mediterranean variant of G6PD deficiency showed a significantly higher level of DNA stand breakage than PBMC of healthy control persons. 
T Efferth ${ }^{*, 1}$, SM SchwarzF ${ }^{2}, J$ Smith $^{2}$ and R Osieka ${ }^{3}$

1 German Cancer Research Center, Im Neuenheimer Feld 280, Heidelberg, Germany

2 Computational Molecular Biophysics, Interdisciplinary Center for Scientific Computing (IWR), University of Heidelberg, Im Neuenheimer Feld 368, Heidelberg, Germany

${ }^{3}$ Medical Clinic IV, Technical University of Aachen, Pauwelsstraße 30, Aachen, Germany

* Corresponding author: T Efferth, German Cancer Research Center, Im Neuenheimer Feld 280, 69120 Heidelberg, Germany. Tel: + 496221 423426; Fax: + 496221 429605; E-mail: t.efferth@dkfz.de

1. Miwa S and Fujii H (1996) Am. J. Hematol. 51: 122-132

2. Pai GS et al. (1980) Proc. Natl. Acad. Sci. USA 77: 2810-2813

3. Beutler E and Vulliamy TJ (2002) Blood Cells Mol. Dis. 28: 93-103

4. Xu W et al. (1995) Blood 85: 257-263

5. Efferth T et al. (2004) Blood 104: 2608

6. Fico A et al. (2004) Cell Death Differ. 11: 823-831

7. Efferth T et al. (1995) J. Mol. Med. 73: 47-49
8. Briehl MM et al. (1997) Oncol. Res. 9: 281-285

9. Müller I et al. (1998) Int. J. Mol. Med. 1: 491-494

10. Taatjes DJ et al. (1998) Curr. Pharm. Des. 4: 203-218

11. Tome ME and Briehl MM (2001) Cell Death Differ. 8: 953-961

12. Tome ME et al. (2001) Cancer Res. 61: 2766-2773

13. Miura Y (2004) J. Radiat. Res. 45: 357-372

14. Spitz DR et al. (2004) Cancer Metast. Rev. 23: 311-322

15. Kahn A et al. (1976) Hum Genet 32: 171-180

16. Efferth T, Osieka R and Beutler E (2000) Blood Cells Mol. Dis. 26: 101-104

17. Beutler $E$ et al. (1991) J. Biol. Chem. 266: 4145-4150

18. Au SWN et al. (2000) Structure 8: 293-303

19. Pandolfi PP et al. (1995) EMBO J 14: 5209-5215

20. Efferth T, Fabry U and Osieka R (2001) J. Leukoc. Biol. 69: 340-342

21. Zhang $X$ et al. (1997) Free Radic. Biol. Med. 23: 980-985

22. Pourzand $C$ and Tyrrell RM (1999) Photochem. Photobiol. 70: 380-390

23. Kawanishi S, Hiraku $Y$ and Oikawa S (2001) Mutat. Res. 488: 65-76

24. Nishigori $C$, Hattori $Y$ and Toyokuni S (2004) Antioxid. Redox Signal. 6: $561-570$

25. Godar DE et al. (1993) Photochem. Photobiol. 57: 1018-1026

26. Mesbah-Namin SA, Nemati A and Tiraihi T (2004) Mutat. Res. 568: 179-185 